workforce (business travellers, expatriates, local nationals).The Council sought to examine the extent of this global epidemic and determine what, if any, processes/practices had been implemented by international organisations to mitigate against mental illness negatively impacting the worker and/or company productivity.

Methods The Council surveyed 344 international organisations representing more than 2.5 million workers in multiple industry sectors.

Results All organisations noted a transition in the profile of their mobile employee from baby boomer to millennial, with 21-percent of their mobile workforce considered 'millennial'. Thirteen percent acknowledged that mental illness, within their mobile workforce, was impacting assignments and/or productivity. Thirty percent admitted to having a process to identify 'at-risk' personnel. Forty-nine percent described the provision of traditional and non-traditional support services to employees to mitigate the impact of mental illness in the workplace.

Discussion Mental health issues endemic to millennials span a wide spectrum from 'unseen' stress that reduces well-being and work satisfaction to presenteeism and poor performance, to workplace incidents and, in the extreme, to disruption and suicide. Organisations are struggling to find the best strategies to engage personnel early without breaking confidentiality. Stigma associated with mental illness remains very significant in many countries/cultures so a unidimensional solution will not suffice. Health 'coaches' have been useful in mitigating against stigma and allowing consultations across borders. Telehealth is an important tool that allows rapid access to a counsellor, when one is not available locally.

\section{PSYCHOSOCIAL RISK FOR SURVIVOR DRIVERS IN WORK-RELATED ROAD TRAFFIC FATALITIES}

A Drummond*, M Codd. University College Dublin, Dublin, Ireland

\subsection{6/oemed-2018-ICOHabstracts. 1603}

Introduction Work-related road-traffic management is recognised as an important means of both occupational and public accident prevention. This area is of concern to road safety, public health, and occupational health and safety regulatory authorities. However, the psychological risk for workers who survive work-related fatal collisions is rarely considered.

Methods A retrospective descriptive study was conducted using data from all coroner road traffic fatality records in the Republic of Ireland, focusing on deaths that occurred in the calendar years 2008 to 2011 inclusive. Data were used to ascertain the prevalence and nature of work-related road traffic fatalities, and to identify work-related accident prevention opportunities.

Results It was ascertained that 23\% $(n=193)$ of all road-traffic fatalities $(n=833)$ were work-related. In $15 \%$ of cases a worker died; however, in $85 \%$ of cases a 'bystander' died and the (worker) driver of the involved vehicle survived. Surviving drivers comprised mostly professional drivers: truck drivers (52\%), public service vehicle drivers (20\%) and van drivers $(12 \%)$. In $27 \%$ of bystander deaths work contributed to the collision, but in the majority the worker driver's actions did not contribute to the collision. Depositions from survivors and witnesses attested to the trauma visited on workers who survived such collisions, however no evidence was found suggesting psychological support for driver survivors.

Discussion Many workers drive for a living and others drive occasionally as part of work. Work-related driving policies tend to focus on ensuring that worker drivers' vehicles and practices are safe. However, little account is taken of the psychosocial risk and the impact of the unsafe driving practices of other road users on survivor drivers, who are party to fatal collisions. These findings have implications for occupational risk assessment and tertiary prevention strategy planning, including post-traumatic counselling, reintegration into work and employee assistance programmes.

\section{EFFECTS OF A WORK-FAMILY INTERVENTION PROGRAM: A PRETEST-POSTTEST STUDY}

${ }^{1} \mathrm{~A}$ Shimazu* ${ }^{2} \mathrm{M}$ Sakka, ${ }^{3} \mathrm{E}$ Obikane, ${ }^{4} \mathrm{M}$ Arima, ${ }^{5} \mathrm{~S}$ Doi, ${ }^{5} \mathrm{~T}$ Fujiwara, ${ }^{6} \mathrm{~T}$ Hasegawa, ${ }^{7} \mathrm{Y}$ Kato, ${ }^{8} \mathrm{~N}$ Iwata, ${ }^{3} \mathrm{~N}$ Kawakami, ${ }^{9} \mathrm{~K}$ Matsudaira, ${ }^{10} \mathrm{~K}$ Shimada, ${ }^{11} \mathrm{M}$ Takahashi, ${ }^{12} \mathrm{M}$ Takahashi, ${ }^{13} \mathrm{M}$ Tokita, ${ }^{14} \mathrm{H}$ Uchiyama, ${ }^{14} \mathrm{I}$ Watai. ${ }^{1}$ Center for Human Social Science, College of Liberal Arts and Science, Kitasato University, Sagamihara, Japan; ${ }^{2}$ Global Nursing Research Centre, Graduate School of Medicine, The University of Tokyo, Tokyo, Japan; ${ }^{3}$ Department of Mental Health, Graduate School of Medicine, The University of Tokyo, Tokyo, Japan; ${ }^{4}$ Office for Gender Equality and Work Life Balance, Support Centre for Students and Female Staff, Tokyo Medical and Dental University, Tokyo, Japan; ${ }^{5}$ Department of Global Health Promotion, Tokyo Medical and Dental University, Tokyo, Japan; ${ }^{6}$ Department of Human Sciences, Taisho University, Tokyo, Japan; ${ }^{7}$ Department of Psychology, School of Human Sciences, Sugiyama Jogakuen University, Aichi, Japan; ${ }^{8}$ Department of Psychology, Hiroshima International University, Higashi-Hiroshima, Japan; ${ }^{9}$ Department of Medical Research and Management for Musculoskeletal Pain, 22nd Century Medical and Research Centre, Faculty of Medicine, The University of Tokyo, Tokyo, Japan; ${ }^{10}$ Department of Social Psychology, Faculty of Sociology, Toyo University, Tokyo, Japan; ${ }^{11}$ National Institute of Occupational Safety and Health, Kawasaki, Japan; ${ }^{12}$ Department of Clinical Psychology, Graduate School of Education, The University of Tokyo, Tokyo, Japan: ${ }^{13}$ Department of Psychiatric and Mental Health Nursing, Graduate School of Nursing, Chiba University, Chiba, Japan; ${ }^{14}$ Department of Nursing, Graduate School of Medicine, Nagoya University, Nagoya, Japan

\subsection{6/oemed-2018-ICOHabstracts. 1604}

Introduction The current study examines the effectiveness of a newly developed work-family intervention program on one's own work-to-family and family-to-work spillovers (both positive and negative) and work-family balance self-efficacy among Japanese dual-earner workers with pre-school child(ren).

Methods The study design was a single-group pre-post comparison. This intervention program was conducted as a pilot study of TWIN (Tokyo Work-life INterface) study III. Participants were eleven workers of a private company in Japan. They were all dualearner workers with pre-school child(ren). The program consisted of seven 45 min sessions with a two-week interval between them. The outcomes were assessed by a questionnaire at baseline (Time 1 ) and post-intervention (Time 2). The paired t-test was conducted as an indicator of intervention effect. Effect sizes were calculated using Cohen's $d$ based on pre-post score differences (Trial registration: UMIN Clinical Trials Registry UMIN000025803).

Results The program showed a medium and favourable intervention effect on work-to-family positive spillover (Cohen's $d=0.53$ ), and small and favourable intervention effects on family-to-work positive spillover (Cohen's $d=0.33$ ) and workfamily balance self-efficacy (Cohen's $d=0.39$ ). Unexpectedly, it showed a significantly small and adverse effect on work-tofamily negative spillover (Cohen's $d=0.33$ ).

Conclusion The newly developed work-family intervention program was found effective in improving work-family positive spillover (both work-to family and family-to work) and 
work-family balance self-efficacy among Japanese dual-earner workers with pre-school child(ren).

\section{DEVELOPMENT OF AN ONLINE INTERVENTION TOOL TO PREVENT EXPOSURE TO WORKPLACE BULLYING}

1,2,3 Whitney Van den Brande*, ${ }^{2,4}$ Elfi Baillien, ${ }^{1,3}$ Tinne Vander Elst, ${ }^{3,5}$ Hans De Witte, 1,6 Lode Godderis. 'Knowledge, Information and Research Centre (KIR, IDEWE Group), Leuven, Belgium; ${ }^{2}$ Research Department Work and Organisation Studies (KU Leuven), Brussels, Belgium; ${ }^{3}$ Research Group Work, Organisational and Personnel Psychology (KU Leuven), Leuven, Belgium; ${ }^{4}$ Department of Pyschosocial Sience, University of Bergen, Norway; ${ }^{5}$ Optentia Research Focus Area (North-West University), Vanderbijlpark Campus, South Africa; ${ }^{6}$ Centre for Environment and Health (KU Leuven), Leuven, Belgium

\subsection{6/oemed-2018-ICOHabstracts. 1605}

Introduction Although the negative impact of workplace bullying has been empirically demonstrated, there is little research on preventive interventions regarding workplace bullying. In reply, the aim of this study is to develop an evidence-based online intervention tool to prevent employees from being exposed to workplace bullying. Given the significant role of coping strategies and self-efficacy in becoming a target of bullying, we aim to develop an online intervention that (1) discourages emotion-focused coping strategies, (2) stimulates selfefficacy, and (3) reduces exposure to workplace bullying.

Methods The online intervention was developed in three steps. First, we developed a short questionnaire by means of Confirmatory Factor Analysis based on a respresentative sample of the Belgian working population $(n=1.068)$. Second, we developed a personal stress profile module by means of Relative Operating Characteristic Analysis. Third, we developed exercises inspired by the principles of Cognitive Behavioural Therapy.

Result The tool developed (=StressBalancer) contains three steps:

- StressBarometer is a questionnaire that measures the most relevant work-related stressors (i.e., workload, job insecurity, role conflict and role ambiguity), emotion-focused coping strategies and self-efficacy.

- My Stress Profile (= Feedback) provides feedback on the employees' use of emotion-focused coping strategies, their level of self-efficacy and their level of stressors. The profile reports whether employees score safe (= green zone), problematic (= orange zone) or very problematic (= red zone) on these variables.

- Training contains exercises that aim to discourage emotionfocused coping strategies and aim to stimulate self-efficacy.

Discussion Studies have been set up to evaluate the online intervention tool by means of an experimental cross-over design.

\section{EXAMINING THE RELATIONSHIP BETWEEN DEPRESSION SYMPTOM PROFILES AND WORK PRODUCTIVITY LOSS}

${ }^{1}$ Carolyn S Dewa*, 'Jeffrey S Hoch, ${ }^{3}$ Karen Nieuwenhuijsen, ${ }^{3}$ Judith K Sluiter. ' University of California, Davis, Department of Psychiatry and Behavioural Sciences, Sacramento, US; ${ }^{2}$ University of California, Davis, Department of Public Health Sciences, Sacramento, US; ${ }^{3}$ Colonel Institute of Occupational Health, Academic Medical Centre, University of Amsterdam, the Netherlands

10.1136/oemed-2018-ICOHabstracts. 1606
Introduction Depression is one of the most prevalent mental disorders in the working population. About 10\% of workers currently have at least one mental disorder; approximately $2 \%-7 \%$ of the workforce experiences depression. There is growing awareness of the social and economic costs of depression and its heavy workplace burden. Recognising the heterogeneity of workers' experiences of depression, there have been suggestions to focus interventions on individual depression symptoms and symptom clusters (i.e., profiles) rather than depression severity alone. Our analyses explore the question, 'What is the relationship between depression symptom profiles and work productivity loss?'

Methods These analyses use a population-based sample of 2219 employed adults living in Ontario, Canada. Based on PHQ-8 items, depression symptom profiles were identified using cluster analysis methods. Four work productivity loss dimensions (WPLD) were examined: (1) limitations handling time, (2) physical limitations, (3) mental-interpersonal limitations and (4) output demands limitations from the Work Limitations Questionnaire. Associations between the WPLD and the symptom profiles were examined using multiple linear regression.

Results Depression symptom profile groups differed in the magnitude of productivity losses. The group experiencing the highest severity across all symptoms had 5\%-20\% more work productivity losses in all four WPLD compared to the other groups. The group with the most difficulty concentrating had $4 \%-13 \%$ greater productivity losses related to mental-interpersonal limitations than other groups. This group along with those who experienced disruption in sleep, energy, and appetite had $8 \%-12 \%$ greater productivity losses related to limitations handling time than other groups.

Discussion Our results suggest workers have different experiences of depression. To assist workers with depression to continue being productive, attention should be paid to the combinations of types of difficulties and specific job characteristics. By understanding the specific challenges, work accommodations can be designed to target specific challenges workers face.

\section{THE EFFECTS OF MEASURABLE OUTCOMES ON THE ACTIVITY OF HEALTH AT WORK PREVENTION SERVICES}

J Munoz*. Université de Brest Bretagne Occidentale LABERS EA 3149 France. Jorge. munoz@univ-brest.fr

\subsection{6/oemed-2018-ICOHabstracts. 1607}

Introduction In France, governments have been developing health at work plans since the early 2000s. The agricultural sector is no exception. In order to set up such a plan, the French agricultural health insurance fund (Caisse Nationale de la Mutuelle Sociale Agricole) has developed a range of managerial processes. These include outcomes to be achieved, which are in turn based on measurable indicators. The aim of the study is to assess the impact of these managerial processes on prevention.

Methods This research is based on non-participant observations that have been conducted within 5 health at work prevention services over a period of 10 months. Observations have been complemented by 2 series of interviews that have been conducted within the above mentioned services $(n=34)$ and at national level $(n=3)$. Two analyses of these interviews 